\title{
Intensity of bacteraemia associated with conservative dental procedures in
} children

\author{
G. J. Roberts, ${ }^{1,2}$ P. Gardner, ${ }^{3}$ P. Longhurst, ${ }^{4}$ A. E. Black, ${ }^{5}$ and V. S. Lucas, ${ }^{6}$
}

\begin{abstract}
Objectives To explore the individual dento-gingival manipulative procedures that together lead to the placement of a restoration and to estimate the associated intensity of bacteraemia.

Patients and methods Healthy children receiving dental treatment under general anaesthesia provided blood samples 30 seconds after one of four dento-gingival manipulative procedures: 1 . Placement of rubber dam, 2. Use of the high speed drill, 3. Use of the slow speed drill, and 4. Placement of matrix band and wedge. Blood cultures were processed to give the percentage prevalence of bacteraemia, the intensity of organisms per millilitre of blood and the identity of the organisms cultured. Results A total of 257 children were recruited to the study. The percentage positive prevalence of blood cultures was baseline $9.3 \%$, rubber dam placement $-31.4 \%$, slow drill $-12.2 \%$, fast drill $-4.3 \%$, and matrix band and wedge $-32.1 \%$. The intensity of bacteraemia was baseline - $1.2 \mathrm{cfu}$, rubber dam placement $1,962 \mathrm{cfu}$, slow drill $-0.3 \mathrm{cfu}$, fast drill $-1.9 \mathrm{cfu}$, matrix band and wedge $-4.8 \mathrm{cfu}$.

Conclusions These data indicate that dento-gingival manipulative procedures comprising a simple dental restoration can lead to a bacteraemia comparable to that from dental extractions. It is suggested that these data may indicate the need for antibiotic prophylaxis for some aspects of conservative dentistry.
\end{abstract}

$\mathrm{B}$ acteraemia resulting from dental extraction is believed to be an important cause of bacterial endocarditis. ${ }^{1}$ It is therefore recommended that patients with congenital or acquired anomalies undergoing tooth extraction should be given prophylactic antibiotics. $^{2}$ This advice is also given by the International Dental Federation. ${ }^{3}$ The guidelines of the Endocarditis Working Party (EWP) of the British Society of Antimicrobial Chemotherapy recommend that antibiotic prophylaxis be provided for extractions, periodontal surgery and scaling. The American Heart Association (AHA) guide-

\footnotetext{
${ }^{1}$ Professor and Head, Department of Paediatric Dentistry, The Eastman Dental Institute for Oral Health Care Sciences, 256 Gray's Inn Road, London WC1X $8 \mathrm{LD} ;{ }^{2}$ Consultant, Maxillofacial and Dental Department, Great Ormond Street Hospital for Children, Great Ormond Street, London WC1N 3JH; ${ }^{3}$ Technical Head, Department of Microbiology, Guy's and St Thomas' Hospital Trust, Lambeth Palace Road, London SE1 7EH; ${ }^{4}$ Consultant, Department of Orthodontics and Paediatric Dentistry, Dental Institute, Guy's Campus, Guy's, King's College and St Thomas' Dental School, St Thomas' Street, London SE1 9RT; ${ }^{5}$ Senior Lecturer and Consultant, Department of Anaesthetics, Great Ormond Street Hospital for Children, Great Ormond Street, London WC1N 3JH; ${ }^{6}$ Clinical Rresearch Fellow, Department of Oral Medicine, The Eastman Dental Institute for Oral Health Care Sciences, 256 Gray's Inn Road, London WC1X 8LD Correspondence to Graham Roberts ${ }^{1}$

REFEREED PAPER

Received 03.02.99; accepted 25.06.99

(C) British Dental Journal 2000; 188: 95-98
}

lines ${ }^{4}$ specifically refer to '...procedures associated with significant bleeding from hard or soft tissues...' as requiring antibiotic prophylaxis. The AHA guidelines provide a table of procedures for which antibiotic prophylaxis is recommended or not recommended. In the 'not recommended' group is 'placement of rubber dam'. The clear implication is that dento-gingival manipulative procedures which form part of the sequence of events that lead to the placement of a dental restoration do not cause a significant bacteraemia and therefore do not need antibiotic prophylaxis. A study carried out to estimate the efficacy of oral amoxycillin in preventing odontogenic bacteraemia indicated that conservative procedures, as well as surgical procedures, could result in a bacteraemia. ${ }^{5}$ There was insufficient detail in this study to identify dento-gingival manipulation during conservative dentistry as a separate cause of bacteraemia from surgical dentistry (extractions and/or dentoalveolar surgery).

The placement of a simple two surface restoration involves a number of discrete dento-gingival manipulations. These are :

\section{Placement of rubber dam \\ 2. Use of the high speed drill \\ 3. Use of the slow speed drill \\ 4. Placement of matrix band and wedge.}

Any one of these procedures will cause displacement of the gingival tissue and/or movement of the tooth in its socket. Bacteraemia may result from such manipulations; intermittent negative and positive pressure in the gingival vasculature, coupled with undetected gingival damage causing plaque bacteria to be drawn into the blood stream.

The data on the percentage prevalence of bacteraemia following conservative dental procedures have been published previously. ${ }^{6}$ The data on intensity of bacteraemia are novel as are the data on plaque and gingivitis indices. The data on percentage prevalence are reproduced here to aid analysis and interpretation of the new data.

The object of this work was to explore the individual dentogingival manipulations which together would comprise those procedures involved in a two surface restoration. This was to determine which, if any, is associated with a bacteraemia.

\section{Patients and methods}

Subjects and procedures

The project was approved by the ethical committees of Great Ormond Street Hospital for Children NHS Trust and Guy's \& St Thomas' Hospital Trust. Written consent was obtained from the parents and also from children considered old enough to understand. The exclusion criteria were the taking of antibiotics within the previous month, known viral carriage and haemorrhagic disorders. All children required general anaesthesia for their dental treatment. Before the general anaesthetic, the patient was 


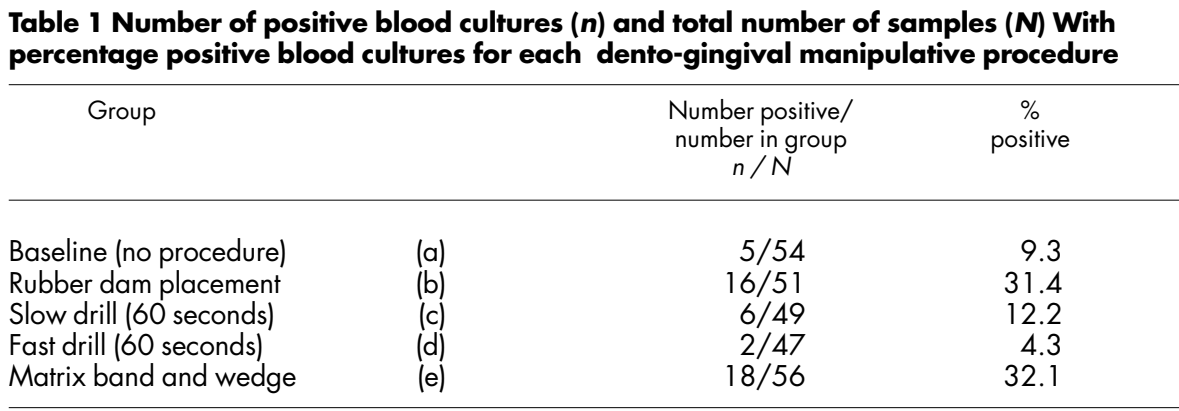

All groups : chi squared $=23.3731 ; \mathrm{df}=4 ; P<0.0001$

Groups b, c, d and e are percentage prevalence data from Roberts et al. $1997^{6}$

\section{Significant differences \\ (a) $\vee$ (b) baseline $v$ rubber dam placement \\ (a) $\vee(e)$ baseline $\vee$ matrix band \\ (b) $v$ (c) rubber dam placement $v$ slow drill \\ (b) $v$ (d) rubber dam placement $v$ fast drill \\ (c) $\vee$ (e) slow drill $v$ matrix band \\ (d) $\vee(e)$ fast drill $v$ matrix band}

\section{Non-significant differences}

(a) $v$ (c) baseline $v$ slow drill

(a) $v$ (d) baseline $v$ fast drill

(b) $\vee$ (e) rubber dam placement $v$ matrix band

(c) $\vee$ (d) slow drill $\vee$ fast drill

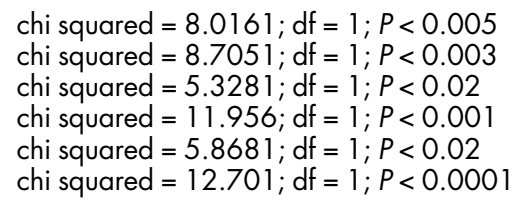

chi squared $=8.0161 ; \mathrm{df}=1 ; P<0.005$

chi squared $=8.7051 ; \mathrm{df}=1 ; P<0.003$

chi squared $=5.3281 ; \mathrm{df}=1 ; P<0.02$

chi squared $=11.956 ; \mathrm{df}=1 ; P<0.001$

chi squared $=5.8681 ; \mathrm{df}=1 ; P<0.02$

chi squared $=12.701 ; \mathrm{df}=1 ; P<0.0001$

chi squared $=0.2401 ; \mathrm{df}=1 ; P<0.5$

chi squared $=0.9755 ; \mathrm{df}=1 ; P<0.3$

chi squared $=0.0073 ; \mathrm{df}=1 ; P<0.9$

chi squared $=2.0047 ; \mathrm{df}=1 ; P<0.2$ assigned to a study group using a random number table. These were: 1 . Baseline (no procedure) 2. Rubber dam placement 3. Slow drill 4. Fast drill 5. Matrix band and wedge. Anaesthesia was induced by intravenous thiopentone or propofol or by inhalation of halothane with nitrous oxide and oxygen. Skin preparation was undertaken with $1 \%$ povidone-iodine solution and an intravenous cannula was inserted into a vein in the antecubital fossa of either arm using aseptic technique.

Once the child was anaesthetised, the distribution of bacterial dental plaque, gingivitis and gingival bleeding were assessed using a modification of the method of O'Leary. ${ }^{7}$ These simplified indices are reproducible in small children. ${ }^{8}$

The procedures are listed in Table 1 and form a sequence of events leading to the completion of a small operation, namely the restoration of a tooth. To ensure that each of these procedures could be studied independently they were performed as a discrete procedure 'out of sequence' immediately after anaesthetic induction, insertion of the naso-tracheal tube and plaque, gingivitis and bleeding assessment. The baseline samples were taken before any dento-gingival manipulation was carried out. (Table 1). One $8 \mathrm{ml}$ blood sample only was taken from each patient 30 seconds $^{9}$ after each of the procedures.

Once these experimental procedures had been completed and the blood sampled for culture, the remaining treatment required was completed.

\section{Bacteriological methods}

Two commercial broth culture systems were used. These were the Bactec 460 radiometric system (Guy's) and the Bactec 760 (GOS) (BACTEC, Beckton Dickinson UK Ltd, Oxford, UK). A 3 ml volume of blood was inoculated into each of the aerobic and the anaerobic bottles. ${ }^{10}$ Bacteria were speciated using standard microbiological methods. Streptococci were speciated using API Strep $20^{11}$ (API Strep 20, Bio Merieux UK Ltd, Basingstoke, UK). A further $1.5 \mathrm{ml}$ was inoculated into the Isolator system vial ${ }^{12}$ ( Isolator Paediatric Merck (UK) Ltd, Poole, Dorset, UK).

Following incubation according to the manufacturer's instructions the results obtained were expressed as the percentage of
Table 2. Colony forming units per/ $\mathrm{ml}$ of blood using the Isolator system

\begin{tabular}{lcccc}
\hline & $n$ & Mean & sd & Range \\
\hline Baseline (no procedure) & 54 & 1.2 & 7.7 & $0-56$ \\
Rubber dam placement & 51 & 1,962 & 14,002 & $0-100,000$ \\
Slow drill (60 seconds) & 49 & 0.3 & 1.5 & $0-8$ \\
Fast drill (60 seconds) & 47 & 1.9 & 13.1 & $0-90$ \\
Matrix band and wedge & 56 & 4.8 & 22 & $0-128$ \\
\hline
\end{tabular}

No significant differences between any of the groups using the Kruskal Wallis Test

Chi squared $=0.239, \mathrm{df}=4, P=0.99$

samples which were positive (Bactec) and the number of colony forming units per millilitre of blood (Isolator). Streptococci were speciated initially using API Strep 20.11

The results are expressed as the percentage of samples which yielded bacteria, the percentage prevalence of positive blood cultures, (Table 1) and the intensity of bacteraemia as the colony forming units per millilitre ( $\mathrm{cfu} / \mathrm{ml}$ ) (Table 2). The organisms isolated were identified to genus level and to species level for oral streptococci (Table 3). ${ }^{13}$ Statistical calculations were made using Stata ${ }^{14}$ and consisted of chi-squared tests for testing differences in proportions, the Shapiro Wilks test for normality and as a consequence the Kruskal Wallis test for non-parametric one way analysis of variance.

\section{Results}

\section{Positive blood cultures}

There were around 50 subjects in each procedure group (Table 1). Anaesthesia was induced by intravenous thiopentone or propofol in 228 patients $(88.7 \%)$ and inhalation of halothane with oxygen and nitrous oxide in a further 29 children (11.3\%), a total of 257 children. Of these 141 were boys (54.7\%) and 116 girls (45.3\%), the mean age was $9 y 1$ mo ranging from $2 \mathrm{y}$ to $19 \mathrm{y} 6 \mathrm{mo}$.

There was a statistically significant difference in the number of positive cultures for the 'rubber dam' and 'matrix band and wedge' groups compared with the 'baseline' group, 'slow drill', and the 'fast 
drill' groups. The statistical significance for all groups is shown in Table 1 and is $P<0.0001$. This significance was then examined in two group comparisons which are either 'significantly different' or 'non-significantly different'. In summary, the placement of a rubber dam or the placement of a matrix band and wedge cause a percentage prevalence of bacteraemia statistically significantly greater than the baseline or the use of either fast or slow drills.

\section{Intensity of bacteraemia}

The intensity of bacteraemia in colony forming units per millilitre of blood is shown in Table 2. The data were non normally distributed when tested with the Shapiro Wilks test. The non-parametric test of analysis of variance by ranks, the Kruskal-Wallis test, showed that there were no significant differences in the intensity of bacteraemia between groups. Most of the lysis centrifugation cultures (Isolator) were negative. The small number of positive cultures from the Isolator were of the order of $1 \times 10^{1}$ to $1 \times 10^{2}$ apart from one sample in the 'rubber dam' group that was $1 \times 10^{5}$.

\section{Micro-organisms isolated}

The organisms isolated are listed in Table 3. and are typical of those associated with bacteraemia of dental origin. . $^{1,5,9}$

\section{Dental indices}

The data for age, plaque index, gingivitis index, and bleeding index were tested for normality using the Shapiro Wilks test and found to be non-normally distributed. Statistical analysis was confined to the non-parametric technique of the two-sample Wilcoxon rank-sum test. ${ }^{14}$ A detailed exploration by each group of samples did not reveal any statistically significant relation between plaque accumulation, gingival inflammation, gingival bleeding and the presence or absence of bacteraemia $(P=0.1$ to $P=0.09)$.

\section{Discussion}

Antibiotic prophylaxis is provided to prevent the development of bacterial endocarditis following odontogenic bacteraemia. Two mechanisms are thought to be involved. First a reduction in the numbers of organisms in the blood and second a reduction in the adhesion of organisms to the non-bacterial thrombotic vegetation. ${ }^{15}$

Currently, the recommendations state that '...procedures causing bleeding...' ${ }^{2}$ are indications for antibiotic prophylaxis. This is in effect limited to scalings and surgical procedures such as single or multiple extractions and dento-alveolar surgery involving the raising of muco-periosteal flaps. ${ }^{3}$ The use of antibiotic prophylaxis for conservative dentistry is not recommended by the American Heart Association, ${ }^{4}$ the Endocarditis Working Party of the British Society for Antimicrobial Chemotherapy ${ }^{2}$ or the Federation Dentaire Internationale. ${ }^{3}$

During the planning phase of this investigation it had been postulated that the vibrations produced by the slow drill or the fast drill might cause fragments of bacterial plaque to be loosened and gain access to the blood stream via damaged gingival blood vessels.

The data presented here suggest this does not happen. The baseline of $9.3 \%$ is similar to the study on dentoalveolar surgery, ${ }^{16}$ which had a baseline value of $11.3 \%$. It is likely that a low level of background bacteraemia is normal as several studies on both adults and children have shown a 'baseline' of the order of $9 \% .^{1}$ This background or baseline feature is a constant finding where sensitive culture techniques are used and is probably the result of small dento-gingival movements in the presence of mild to moderate gingivitis occurring throughout each and every day.

Nevertheless, in this study the placement of a rubber dam and a matrix band with a wedge, procedures frequently undertaken during routine conservative dentistry, resulted in bacteraemia comparable with that encountered following tooth extraction. ${ }^{6}$ In
Table 3 Organisms isolated from blood cultures following conservative dental procedures in children ( $n=$ number of isolates)

\begin{tabular}{ll}
\hline Bactec & Bactec \\
aerobic & anaerobic
\end{tabular}

\begin{tabular}{|c|c|c|c|}
\hline Baseline (no procedure) & $n$ & Baseline (no procedure) & $n$ \\
\hline
\end{tabular}

Rubber dam placement n Rubber dam placement $n$

\begin{tabular}{ll} 
S. sanguis & 4 \\
S. mitis & 4 \\
'Viridans' streptococci & 5 \\
Gram positive bacilli & 1 \\
Neisseria spp. & 2 \\
& \\
Slow drill (60 seconds) & 2 \\
\hline S. sanguis & 1 \\
S. mutans & 2 \\
Coagulase negative staphylococci &
\end{tabular}

Coagulase negative staphylococci 2

S. mitis

S. sanguis 1

'Viridans' streptococci 4

Neisseria spp.

Diphtheroids

Micrococcus spp.

Slow drill (60 seconds)

\begin{tabular}{|c|c|c|c|}
\hline Fast drill (60 seconds) & $n$ & Fast drill (60 seconds) & $n$ \\
\hline & & S. sanguis & 1 \\
\hline
\end{tabular}

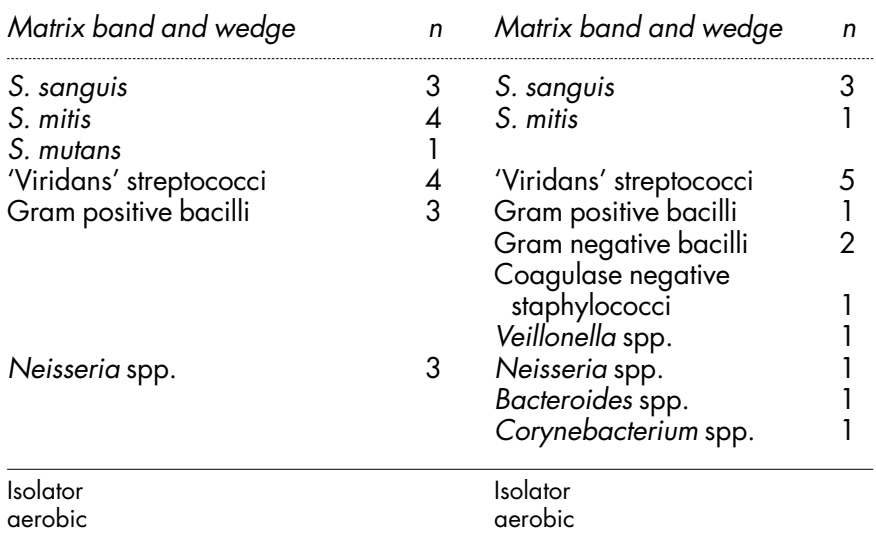

Baseline (no procedure) $n$ Baseline (no procedure) $n$

Streptococcus species $\quad 2 \mathrm{Nil}$
Coagulase negative staphylococci 1

\begin{tabular}{lclc} 
Rubber dam placement & $n$ & Rubber dam placement & $n$ \\
\hline Listeria spp & 1 & Corynebacterium hoffmani & 1 \\
S. epidermidis & 1 & S. epidermidis & 1 \\
Viridans streptococci & 3 & &
\end{tabular}

\begin{tabular}{|c|c|c|c|}
\hline Slow drill (60 seconds) & & Slow drill (60 seconds) & $n$ \\
\hline Coagulase negative staphylcocci & 1 & Gram negative bacilli & 1 \\
\hline Fast drill (60 seconds) & $n$ & Fast drill (60 seconds) & $n$ \\
\hline S.sanguis & 1 & & \\
\hline Nil matrix band and wedge & $n$ & Matrix band and wedge & $n$ \\
\hline $\begin{array}{l}\text { S.mitis } \\
\text { Coagulase negative staphylococci } \\
\text { Bacillus spp. }\end{array}$ & $\begin{array}{l}2 \\
1 \\
1\end{array}$ & Gram positive bacilli & 1 \\
\hline
\end{tabular}


addition, the highest intensity of bacteraemia follows the two procedures with the greatest degree of dentogingival manipulation namely rubber dam placement and matrix band with wedge. Indeed, the very high value for rubber dam placement is perhaps the result of the interproximal rubber being forced down between the teeth and at the same time pushing the dental plaque ahead of it and then squashing it onto the inflamed gingivae.

On reflection these findings are not unexpected as considerable manipulation of the gingival tissue takes place during rubber dam placement and matrix band and wedge placement. Although it was not tested it would be reasonable to expect that matrix band placement on its own, particularly when it causes gingival bleeding, would also result in bacteraemia.

The reasons for the passage of dental plaque bacteria into the blood stream during dento-gingival manipulations are not understood. During extractions it has been suggested that rocking the tooth in its socket prior to removal results in alternating negative and positive pressure in the gingival and periodontal vasculature, so that bacterial plaque fragments dislodged from the tooth surface are drawn into the blood stream. In the case of rubber dam clamp and gingival wedge placement, it is probable that bacterial plaque fragments are inoculated into the deeper tissues and thence into the blood stream via the traumatised gingival and periodontal blood vessels. This inoculation is likely to be facilitated if the gingival tissue is inflamed and in this study, bleeding was associated with rubber dam placement and the use of a matrix band and wedge.

The data on plaque and gingivitis are limited but did not show any clear relationship between the indices and the presence or absence of bacteraemia. This is disappointing as there is anecdotal belief of a presumed relationship. It is difficult to explain this apparent lack of association but it may simply be the difference in methods of measurement ie gross deposits of plaque on a 0 or 1 assessment compared with individual colony forming units from the blood cultures.

The results of this study suggest that the AHA guideline that antibiotic prophylaxis for rubber dam placement is not recommended may need to be re-examined. Our data provide evidence that aspects of conservative dental procedures (rubber dam and matrix band with wedge placement) may result in significant bacteraemia which should be covered by antibiotic prophylaxis. The data presented here have implications for treatment planning as it would be of considerable benefit to the child to have as much treatment carried out under the protection of a single dose of antibiotic prophylaxis. The use of chlorhexidine mouthrinse has been recommended ${ }^{17}$ but may not be feasible in young children who may refuse the use of the unpleasant tasting formulation of chlorhexidine currently available.

These new data have implications for the advice related to the use of antibiotic prophylaxis related to dental treatment in children with congenital or acquired heart disease. Is the clear existence of a significant bacteraemia following conservative dentistry procedures justification for antibiotic prophylaxis? A recent paper examined the relationship between the risk factors for endocarditis and showed that in a case control study that dental treatment was not a risk factor for infective endocarditis. ${ }^{18}$ Whether it is yet time to abandon antibiotic prophylaxis for dental treatment or whether further evidence is required is a matter for the Endocarditis Working Party and similar advisory bodies at national and international level.

The authors gratefully acknowledge the assistance of staff in the departments of microbiology at St Thomas' Hospital and Great Ormond Street Hospital for Children.

1 Everett E D, Hirschmann J V. Transient bacteremia and endocarditis prophylaxis. A review. Med 1977; 56: 61-77.

2 Endocarditis Working Party of the British Society for Antimicrobial Chemotherapy. Recommendations for endocarditis prophylaxis. J Antimicrob Chemoth 1993; 31: 437-438.

3 International Dental Federation. Guideline for antibiotic prophylaxis of infective endocarditis for dental patients with cardiovascular disease. Int Dent J 1987; 37: 235-236.

4 Dajani A S, Taubert K A, Wilson W et al. Prevention of bacterial endocarditis. Recommendations by The American Heart Association. JAMA 1997; 277: 1794-1801.

5 Roberts G J, Radford P, Holt R. Prophylaxis of dental bacteraemia with oral amoxycillin in children. Br Dent J 1987; 162: 179-182.

6 Roberts G J, Holzel H, Sury M R J, Simmons N A, Gardner P, Longhurst P. Dental bacteraemia in children. Ped Cardiol 1997; 18: 24-27.

7 O'Leary T J, Drucker D B, Naylor J E. The plaque control record. J Periodontol 1972; 43: 38-42.

8 Franco E, Saunders C, Roberts GJ, and Suwanprasit H. Dental disease, caries related microflora and salivary IgA of children with severe congenital cardiac disease. Pediatr Dent 1996; 18: 228-235.

9 Roberts G J, Gardner P, Simmons N A. Optimum sampling time for detection of odontogenic bacteraemia in children. Int J Cardiol 1992; 35 : 311-315.

10 Daley C, Lim I, Modra J, Wilkinson I. Comparative evaluation of nonradiometric BACTEC and improved oxoid signal blood culture systems in a clinical laboratory. J Clin Microbiol 1990; 28: 1586-1590.

11 Waitkins S A, Anderson D R, Todd F K. An evaluation of the API-STREP identification system. Med Lab Sciences 1981; 38: 35-39.

12 Brannon P, Kiehn T E. Large scale clinical comparison of the lysis centrifugation and radiometric systems for blood culture. J Clin Microbiol 1985; 22: 951-954.

13 Wiley R A, and Hardie J M. Emmerson A M H, Hawkey P M, Gillespie S H, (eds). Principles and Practice of Clinical Bacteriology. First ed. Chichester: John Wiley and Sons; 1997; 1a, alpha haemolytic and non-haemolytic streptococci. pp 3-35.

14 STATA. Stata Reference Manual: release 3. 5 ed. Santa Monica, California: Computing Resource Centre; 1992.

15 Glauser M P, Bernard J P, Moreillon P, Francioli P. Successful single-dose amoxicillin prophylaxis against experimental streptococcal endocarditis : evidence for two mechanisms of protection. J Infect Dis 1983; 147: 568-575.

16 Roberts G J, Watts R, Longhurst P, Gardner P. Bacteremia of dental origin and antimicrobial sensitivity following oral surgical procedures in children. Pediatr Dent 1998; 20: 28-36.

17 MacFarlane T W, Ferguson M M, and Mulgrew C J. Post-extraction bacteraemia: role of antiseptics and antibiotics. Br Dent J 1984; 156: 179-181.

18 Strom B L, Abrutyn E, Berlin J E et al. Dental and cardiac risk factors for infective endocarditis. Ann Intern Med 1998; 129: 761-769. 